\title{
Polar UVI images to study steady magnetospheric convection events: Initial results
}

\author{
Anna D. DeJong and C. Robert Clauer \\ Atmospheric Oceanic and Space Science, University of Michigan, Ann Arbor, Michigan, USA \\ Received 26 August 2005; revised 10 October 2005; accepted 25 October 2005; published 16 December 2005.
}

[1] During periods of steady enhanced magnetospheric driving, it may be possible for the reconnection rate on the nightside to adjust to match the reconnection rate on the dayside. When this occurs, it is referred to as a steady magnetospheric convection event or SMC. This balance of reconnection rates is reflected by unchanging open flux in the lobes, which can be inferred using the open-closed boundaries on auroral images. We use Polar UVI data to determine this boundary and calculate the area inside the polar cap. The temporal changes in this area are used to determine the degree of balance between the dayside and nightside reconnection rates. Thus far, we have identified 22 SMC events. We relate these events to past SMC studies. The preliminary results presented here support many past conclusions while also contradicting others. Citation: DeJong, A. D., and C. R. Clauer (2005), Polar UVI images to study steady magnetospheric convection events: Initial results, Geophys. Res. Lett., 32, L24101, doi:10.1029/ 2005 GL024498.

\section{Introduction}

[2] When the interplanetary magnetic field (IMF) is southward (negative Bz) and steady for an extended period of time, the magnetosphere can go for more than 3 hours without substorm expansion signatures. When this occurs, the convection in the magnetosphere is thought to be stable and we have what is referred to as steady magnetospheric convection or SMC. While an absence of substorm signatures is a good indicator of an SMC, it lacks physical meaning for a definition. If magnetospheric convection is truly steady, then the reconnection rates on the dayside and nightside must balance [Cowley and Lockwood, 1992; Siscoe and Huang, 1985]. During such a period, the flux of open field lines in the lobes must remain constant. Since these field lines map down to the interior of the auroral oval, its poleward boundary provides an approximate measure of the open flux in the lobes. If the area inside this boundary remains constant, then the amount of open field lines most likely remains steady during that time. This implies that the reconnection rates balance. Thus, we have steady magnetospheric convection.

[3] Although others have used the aurora and the poleward auroral boundary to study SMCs [Sergeev et al., 2001; Yahnin et al., 1994], it has yet to be used as a selection criteria. The area inside this boundary is referred to as polar cap area or PC area. We classify an event as steady magnetospheric convection if it satisfies the following: PC area remains steady for longer than 3 hours, AE is greater than $200 \mathrm{nT}$, there are no other substorms signatures.

\section{Identifying Steady Magnetospheric Convection Events}

[4] As stated previously, in order to classify an event as an SMC we first need to determine the poleward boundary of the auroral oval. This is done using Polar UVI images in the Lyman-Birge-Hopfield long (LBHl) spectrum. A threshold of auroral luminosity of 4.3 photons $/ \mathrm{cm}^{2} / \mathrm{s}$ (approx. 130 Rayleighs) is used to determine the boundary [Baker et al., 2000]. When part of the oval is missing due to orbital motion, the rest of the boundary is extrapolated using a curve-fit to the visible boundaries [Brittnacher et al., 1999]. Once the boundary is determined, the area inside is calculated for the polar cap.

[5] If we are to look for balanced reconnection rates, or steady PC area, we must first understand what happens when reconnection rates do not balance. During the growth phase of a substorm, reconnection occurs on the dayside when the IMF turns southward. This causes a build up of open flux on nightside and an increase in the PC area. At the onset of the expansion phase there is a rapid increase in reconnection rate on the night side, causing the aurora to brighten near midnight and to expand polarward. This in turn causes a drop in the PC area. The substorm at 0310 UT in Figure 1c shows a drop from $15 \times 10^{6} \mathrm{~km}^{2}$ to $10 \times 10^{6} \mathrm{~km}^{2}$ in about 45 minutes. The recovery phase can also be seen here, as the PC area starts to grow back to its original size. For a more detailed analysis of polar cap boundaries during substorms, we refer the reader to Milan et al. [2003] and Kamide et al. [1999].

[6] Since our polar cap boundary is only an approximation of the open flux, we are more interested in the temporal changes in the PC area than its value. The time variations in the PC area should accurately monitor the changes in the open field lines. Our boundary method shows that, during a substorm, the PC area drops from $20 \%$ to $30 \%$ in one hour or less. Thus, we adopt the criterion that $\mathrm{PC}$ area variation must be less than $10 \%$ within a 1 hour interval during the SMC event. However, small variations, falling well within our systematic error of $10 \%$, do occur due to changes in Polar's coverage and the boundary threshold. Sergeev et al. [1996] found that SMCs begin and end with substorm signatures. Many of our events also show this to be true. Thus, this methodology allows us to differentiate between the substorm signatures and the SMC. In order to avoid including recovery phases of substorms in our analysis, which sometimes have a steady PC area, we use a 3 hour minimum. Finally, we ensure that only active periods are measured, by requiring that $\mathrm{AE}$ be greater than $200 \mathrm{nT}$. 


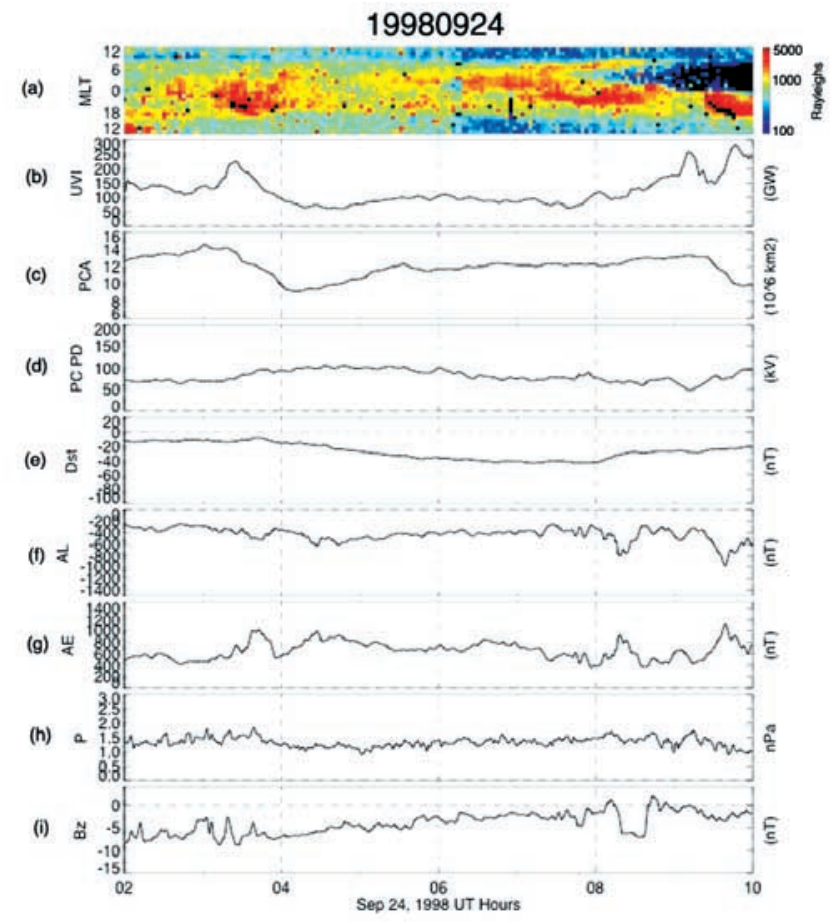

Figure 1. A stack plot of data from Polar UVI and magnetospheric indices from the SMC on September 24, 1998. The panels from top show (a) MLT UT map of the maximum photon flux, (b) auroral energy flux, (c) Polar cap area, (d) Cross polar cap potential difference, (e) AL, (f) AE, (g) Dst, (h) Solar wind dynamic pressures, and (i) IMF Bz.

[7] Figure 1 is a stack plot of data from an SMC that occurred on September 24th 1998. Figure 1a is a map of the maximum intensity (in Rayleighs) in Magnetic Local Time (MLT) versus Universal Time (UT). Figure $1 \mathrm{~b}$ is the UVI auroral power calculated from the LBHl [Brittnacher et al., 1997]. Figure $1 \mathrm{c}$ is the polar cap area in $10^{6} \mathrm{~km}^{2}$. The cross polar cap potential difference (PCPD), AL and AE are shown in Figures 1d, 1f, and 1g, respectively. They are derived by assimilative mapping of ionospheric electrodynamics (AMIE) which has been applied to approximately 150 ground based high latitude magnetometer records [Ridley and Kihn, 2004]. The $\mathrm{D}_{\text {st }}$ (Figure 1e) is calculated from mid-latitude magnetometer stations [Clauer and McPherron, 1974]. The solar wind dynamic pressure and the IMF Bz, Figures $1 \mathrm{~h}$ and 1i, are from the ACE satellite and have no time delays.

[8] Figure 2 shows an MLT-UT (Magnetic Local Time Universal Time) map of magnetic perturbations made with approximately 24 mid-latitude magnetometer stations. The contours are drawn every $5 \mathrm{nT}$, where the red contours show a decrease in Earth's magnetic field and the blue contours show an increase. In these maps, we look for localized red regions to indicate partial ring current development during storms. Similarly, a small blue region on the nightside illustrates a mid-latitude positive bay that is a signature of a substorm current wedge. For details on the mapping process see Clauer and McPherron [1974].

[9] As stated previously, many of our SMCs both begin and end with substorm signatures, as the event in Figures 1 and 2 illustrate. The SMC occurs from 0530 UT to 0920 UT
(Figure 1c) where the PC area is the most steady. The initiating substorm is a double onset with the first onset at 0310 UT. The first onset can be seen as a blue area at 2100 MLT and 0300 UT in Figure 2 while the second onset occurs at 0300 MLT 20 minutes later. This substorm is also noticeable in the first three panels of Figure 1. There is a brightening in Figure 1a and a peak in the auroral power in Figure 1b. The PCPD shows a small increase at this time along with a very small decrease in AL. After the recovery phase of the initiating substorm at $0530 \mathrm{UT}$, the PC area remains steady until the substorm at $0920 \mathrm{UT}$. The only data that is not very steady during the SMC are the AL and AE indices. The peaks at 0815 UT are most likely caused by an intensification of the westward electrojet, which is also seen as a small brightening in the auroral oval. This brightening can be seen in Figure 1a but not in Figure 1b. Since there is no change in the PC area or other data we believe this to be a pseudo-breakup rather than a substorm. During the concluding substorm, we once again see a brightening in Figure 1a. There are also peaks in the PCPD, AL and AE traces. The PC area also begins to decrease but Polar imaging coverage is lost around 1000 UT. Hence, we cannot truly measure the change in the PC area during the final substorm. Figure 2 also supports the interpretation of a substorm onset at 0920 UT.

[10] Due to the Polar satellite's orbital coverage and the length of some of our events, we do not always have imaging for the full event. When this occurs, we look at other data (AL, LANL SoPa, midlatitude magnetometers and PCPD) to determine the duration of the SMC. Frequently, there is a substorm in the data to indicate the ending or beginning of the SMC. However, instances occur when the signatures in the data weaken to quiet levels before a substorm is indicated.

\section{Initial Results}

[11] Thus far, we have identified a total of 22 SMC events from Polar UVI images taken during the years 1997 to 2001. There are not enough events yet to claim unambiguous statistical results. However, initial results show that only one occurs during a moderate storm $\left(\mathrm{D}_{s t}<\right.$

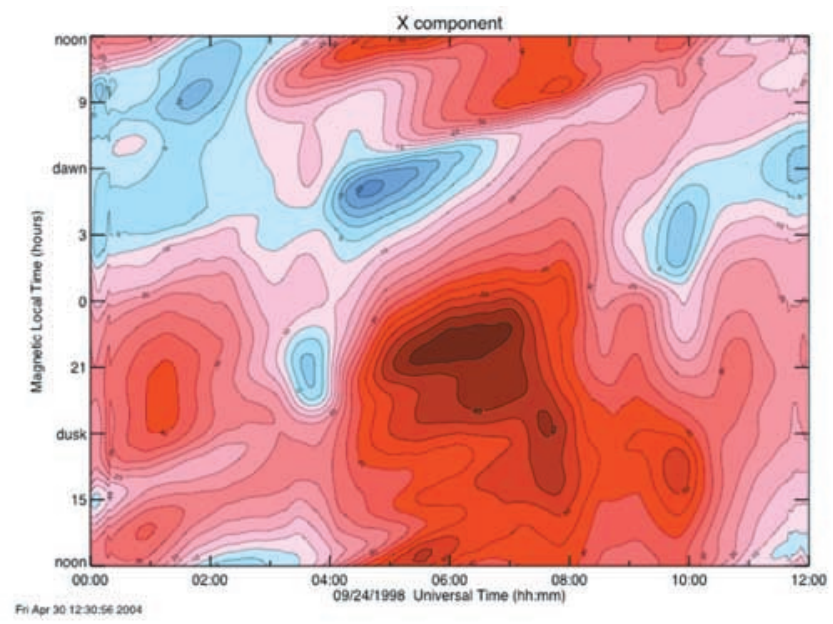

Figure 2. An MLT-UT map of mid-latitude magnetic perturbations for the SMC on Sept. 24, 1998. 


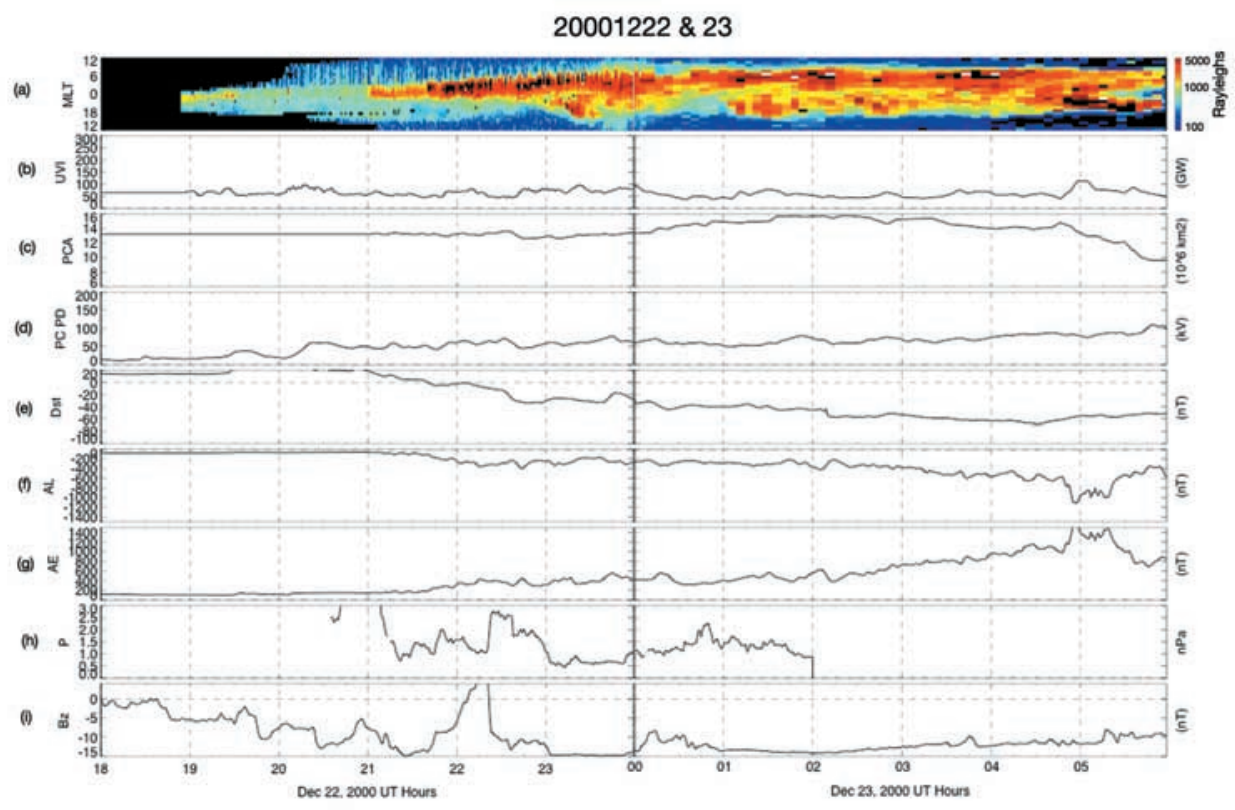

Figure 3. The same stack plot as in Figure 1 only of the SMC on December 22nd and 23rd of 2000.

$-100 \mathrm{nT})$. Most of them occur during weaker storms at around a $\mathrm{D}_{s t}=-50 \mathrm{nT}$. The weakest $\mathrm{D}_{s t}$ of the events is $-15 \mathrm{nT}$. These findings are similar those found by Sergeev et al. [1996], where the events ranged from a $\mathrm{D}_{s t}$ of $-61 \mathrm{nT}$ to $-4 \mathrm{nT}$. Along with $\mathrm{D}_{s t}$, we are also interested in the IMF/ Solar wind inputs during these events. Since the IMF $\mathrm{Bz}$ tends to be steady during an SMC, we took the average the IMF Bz for each event. The ensemble mean of the average Bz's is $-5 \mathrm{nT}$ and the median is $-4 \mathrm{nT}$. This is in agreement with past studies that found an average $\mathrm{Bz}$ of $-4 \mathrm{nT}$ [Sergeev et al., 1996; O'Brien et al., 2002]. When more events are found, solar wind dynamic pressure and IMF By will be studied to see if there are any correlations with SMC events.

[12] Not all SMC events appear to be the same. We have identified various types of SMCs based upon their temporal changes in PC area. Two of our events have a long period of steady PC area followed by a growth of approximately $30 \%$ over 3 hours, eventually ending with a substorm. During these events the convection is fairly steady with a slow flux buildup in the tail. These events could also be considered very slow growth phases. We are hoping to find more events similar to these so that they may be studied in greater detail.

[13] According to Sergeev et al. [1996] all SMCs begin and end with substorms. We have not found this to be true. Of our 22 events, only 18 both begin and end with substorms. Two events only begin with a substorm, having no substorm signatures at the end. Conversely, three events show no substorm signatures at the beginning. The magnetosphere seems to slowly ramp up to higher activity with no substorm. One of these events is shown in Figure 3. This SMC occurred on December 22nd and 23rd of 2000. It starts at 2200 UT on the 22nd when AE becomes greater than $200 \mathrm{nT}$. It then ends at $0445 \mathrm{UT}$ on the 23rd when there are substorm signatures seen in AL, PC area, and UVI data of Figure 3. At the beginning of the SMC the aurora brightens slowly without distinct signatures of a substorm expansion. The cross polar cap potential difference (PCPD), as determined from AMIE, slowly increases and then remains steady with no sharp changes that would indicate a substorm. AL and AE similarly show no substorm signatures in the beginning of the SMC. Note it takes almost 7 hours for AE to go from $100 \mathrm{nT}$ to $1000 \mathrm{nT}$. The PC area increases slowly during the first 3 hours and then remains steady from 0100 UT until 0445 UT. The aurora then moved poleward at expansion onset and caused a drop in the polar cap area. Figure 4 shows an MLT-UT map similar to Figure 2, however the contours here are every $7 \mathrm{nT}$. This particular event has a strong partial ring current, so the substorm at the end of the event shows up as a pink region around 0500 UT and 0130 MLT. This plot also indicates that there is no substorm at the beginning of this

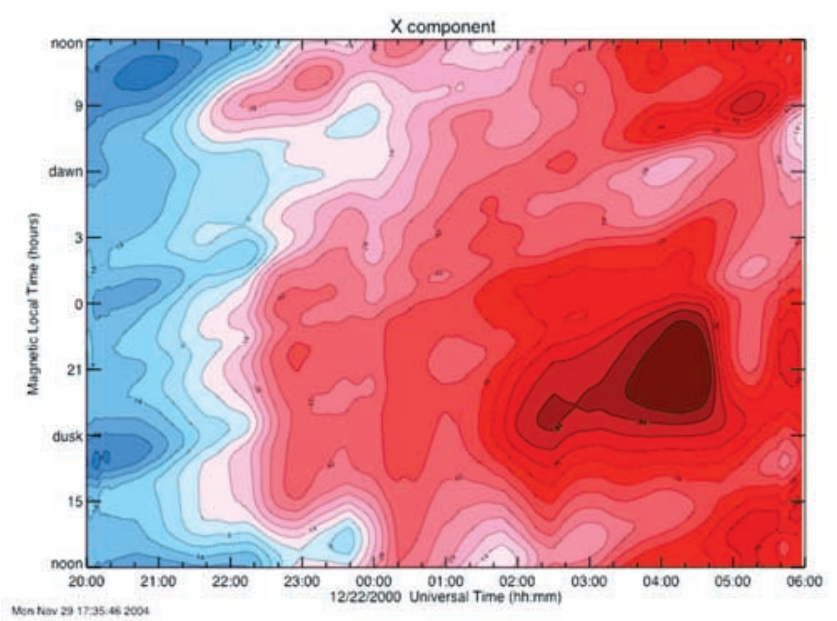

Figure 4. An MLT-UT map of mid-latitude magnetic perturbations for the SMC on Dec. 22nd and 23rd of 2000. 
event. Note the small blue regions around 2330 UT are too close to noon to be caused by a substorm current wedge.

\section{Discussion}

[14] Recently O'Brien et al. [2002] did a statistical study of SMCs, using only AL and AE to define their events. While this allowed them to find many events for the statistical investigation, this selection method has some unwanted consequences. Their minimum time limit was 90 minutes in contrast to our requirement of at least 3 hours, thus permitting substorm recovery phase intervals to enter into their analysis. They also inflict a limitation of AL(t) $\mathrm{AL}(\mathrm{t}-1 \mathrm{~min}) \geq-25 \mathrm{nT}$ that only allows them to find weaker events. We have found that events with larger AL signatures can also have larger fluctuations. Also, as can be seen in Figure 1 at 0815 UT, we get a large drop in AL during our pseudo-breakup. Since we have found pseudo-breakups during some of our events, it is possible that O'Brien et al. [2002] are missing events or cutting some events short. Unfortunately, we cannot compare our data at this point since their data is from 1978-1988 and ours is much later.

[15] As stated in the previous section, we have periods of steady magnetospheric convection that contain pseudobreakups or localized auroral activity that does not expand into a full substorm [McPherron, 1991]. Ten out of our 22 events show evidence of at least one pseudo-breakup during the SMC. We are not quite sure yet what role these pseudobreakups play in steady magnetospheric convection, but we believe that they might be a way of releasing energy on a small scale in order to keep convection steady on a large scale. There have been studies of pseudo-breakups during quiet times [Fillingim et al., 2000], but they have yet to be studied during SMCs. Sergeev et al. [2001] found brightenings in the aurora associated with streams and fast plasma flows, but does not refer to them as pseudo-breakups. Yahnin et al. [1994] also saw auroral brightenings and small poleward boundary movement, but did not go into much detail. A more in depth study on pseudo-breakups and their role in magnetospheric dynamics, particularly during SMC intervals, is required.

[16] The final piece to be studied is that of solar wind/ IMF drivers. Recent studies have shown that the solar wind/ IMF drivers for SMCs are very similar to those seen during sawtooth events [Borovsky, 2004]. So the question to resolve is what allows the mode of magnetospheric convection to go from steady to sawtooth oscillations.

[17] Ultimately, we believe that using a definition of SMCs that is based on the physical state of the magnetosphere will permit a more robust investigation of SMC events.
[18] Acknowledgments. This research was supported by a NASA GSRP through MSFC, and NASA grants NAG5-12040 and NAG512176. Thanks to Aaron Ridley for the AMIE data and thank you to Jo Baker for use of his codes. We thank George Parks, PI for UVI and his team for their efforts to provide the high quality UVI data base, and for software that forms a foundation for our analysis software.

\section{References}

Baker, J. B., C. R. Clauer, A. J. Ridley, V. O. Papitashvili, M. J. Brittnacher, and P. T. Newell (2000), The nightside polarward boundary of the auroral oval as seen by DMSP and the Ultraviolet Imager, J. Geophys. Res., 105, 21,267-23,280.

Borovsky, J. E. (2004), Global sawtooth oscillations of the magnetosphere, Eos Trans. $A G U, 85(47)$, Fall Meet. Suppl., Abstract SM23B-04.

Brittnacher, M., R. Elsen, G. Parks, L. Chen, G. Germany, and J. Spann (1997), A dayside auroral energy deposition case study using the Polar Ultraviolet Imager, Geophys. Res. Lett., 24, 991-994.

Brittnacher, M., M. Fillingim, G. Parks, G. Germany, and J. Spann (1999), Polar cap area and boundary motion during substorms, J. Geophys. Res., $104,12,251-12,262$.

Clauer, C. R., and R. McPherron (1974), Mapping the local time-universal time development of magnetospheric substorms using mid-latitude magnetic observations, J. Geophys. Res., 79, 2811-2820.

Cowley, S. W. H., and M. Lockwood (1992), Excitation and decay of solar wind-driven flow in the magnetophere-ionosphere system, Ann. Geophys., 10, 103-115.

Fillingim, M. O., G. K. Parks, L. J. Chen, M. Brittnacher, G. A. Germany, J. F. Spann, D. Larson, and R. P. Lin (2000), Coincident POLAR/UVI and WIND observations of pseudobreakups, Geophys. Res. Lett., 27, $1379-1382$

Kamide, K., S. Kokubun, L. F. Bargatze, and L. A. Frank (1999), The size of the polar cap as an indicator of substorm energy, Phys. Chem. Earth, 24, 119-127.

McPherron, R. L. (1991), Physical processes producing magnetospheric substorms and magnetic storms, in Geomagnetism, vol. 4, edited by J. A. Jacobs, 593 pp., Elsevier, New York.

Milan, S. E., M. Lester, S. W. H. Cowley, K. Oksavik, M. Brittnacker, R. A. Greenwald, G. Sofko, and J. P. Villain (2003), Variations in the polar cap area during two substorm cycles, Ann. Geophys., 21, 1121-1140.

O'Brien, T. P., S. M. Thompson, and R. L. McPherron (2002), Steady magnetospheric convection: Statistical signatures in the solar wind and AE, Geophys. Res. Lett., 29(7), 1130, doi:10.1029/2001GL014641.

Ridley, A. J., and E. A. Kihn (2004), Polar cap index comparison with AMIE cross polar cap potential, electric field and polar cap area, Geophys. Res. Lett., 31, L07801, doi:10.1029/2003GL019113.

Sergeev, V. A., R. J. Pellinen, and T. I. Pulkkinen (1996), Steady magnetospheric convection: A review of recent results, Space Sci. Rev., 75, $551-$ 604.

Sergeev, V. A., M. V. Kubyshkina, K. Liou, P. T. Newell, G. Parks, R. Nakamura, and T. Mukai (2001), Substorm and convection bay compared: Auroral and magnetotial dynamics during convection bay, J. Geophys. Rev., 106, 18,843-18,856.

Siscoe, G. L., and T. S. Huang (1985), Polar cap inflation and deflation, J. Geophys. Rev., 90, 543-547.

Yahnin, A., et al. (1994), Features of steady magnetospheric convection, J. Geophys. Res., 99, 4039-4051.

C. R. Clauer and A. D. DeJong, Atmospheric Oceanic and Space Science, University of Michigan, Space Science Research Building, Ann Arbor, MI 48109, USA. (rclauer@umich.edu; dejonga@umich.edu) 